\title{
Grammatische Terminologie im Kontrast. Einige Überlegungen aus der Sicht des DaF-Unterrichts in Italien
}

\author{
Barbara Ivančić (Bologna)
}

\begin{abstract}
The article deals with the metalinguistic terminology which characterizes the grammars of the German language used in the field of German as a foreign language (Deutsch als Fremdsprache) in Italy. Starting from the basic distinction between terminological equivalence and terminological divergence, such as postulated by Kleineidam, the investigation concerns two specific fields, the terminology of, respectively, word classes and phrasal constituents, which is compared with the terminology used in teaching the native language.
\end{abstract}

\section{$1 \quad$ Einführung}

Der vorliegende Beitrag hat die metasprachliche Terminologie zum Gegenstand, die in Grammatiken für den Deutsch-als-Fremdsprache-Unterricht ${ }^{1}$ verwendet wird. Das Untersuchungskorpus besteht dabei aus den Grammatikbüchern, die laut einer an italienischen Schulen und Universitäten durchgeführten empirischen Studie in der Unterrichtspraxis eine häufige Verwendung finden (cf. Ivančić 2003). Es handelt sich insbesondere um die folgenden Grammatiken:2 Tipps (Difino/Fornaciari 1999), Deutsch Klipp und Klar (Difino/Fornaciari 2001), Grammatica tedesca con esercizi (Dreyer/Schmitt 1993/1999), Lehr- und Übungsbuch der deutschen Grammatik (Dreyer/Schmitt 1985/1996), Klipp und Klar (Fandrych/Tallowitz 2000), Deutsche Grammatik (Bruno/Franch 1994), Dies und Das (Weerning/Mondello 2000), Arbeitsgrammatik (Seiffarth/Medaglia 1998), Grammatica di base della lingua tedesca (Reimann 1998), Übungsgrammatik DaF für Fortgeschrittene (Hall/Scheiner 1997) und Grammatica descrittiva della lingua tedesca (Saibene 2002). Die meisten dieser Grammatiken wenden sich - wie bereits den Titeln zu entnehmen ist - explizit an italienischsprachige Lernende des Deutschen. Im Hinblick darauf soll der Aspekt der Terminologie vor allem im interlingualen Vergleich untersucht werden, d.h. durch eine Gegenüberstellung der grammatischen Terminologie im Deutschen und im Italienischen.

Es stellt sich zunächst die Notwendigkeit, Grammatikbücher dieser Art näher einzuordnen.

\section{Begriffliche Klärung: didaktische Grammatik-Fremdsprachengrammatik}

Grammatiken, die für den Sprachunterricht konzipiert werden, werden in der Fachliteratur häufig als didaktische Grammatiken bezeichnet (cf. Götze 2001, Helbig 2001). Wenngleich der Begriff selbst sehr umfassend ist und daher in unterschiedlicher Weise verstanden werden kann, beruht er gleichzeitig auf einer grundsätzlichen Differenzierung zwischen linguistischen und didaktischen Grammatiken, so wie sie im deutschsprachigen Raum vor allem Helbig

\footnotetext{
${ }^{1}$ Im Folgenden DaF-Unterricht.

${ }^{2}$ Die Grammatiken werden nach der Häufigkeit ihrer Nennung in der Umfrage zitiert. Das Jahr bezieht sich auf die zur Zeit der Untersuchung geläufige Ausgabe; in einigen Fällen sind inzwischen auch neuere Versionen erschienen.
} 
vertreten hat (cf. Helbig 1981: 56-62), über die weitgehender Konsens herrscht. Als linguistisch gelten dabei jene Grammatiken, "die eine vollständige und explizite Beschreibung der objektiv der Sprache selbst innewohnenden Regeln darstell[en]", während das Attribut didaktisch auf die Verfahren der "Auswahl und Adaption von dieser LG [= linguistischen Grammatik]" für die Zwecke des Fremdsprachenunterrichts hinweist (Helbig 2001: 103). Unter didaktischer Grammatik wird demnach ganz allgemein ein lehrwerkunabhängiges (oder auch lehrwerkbegleitendes) Grammatikbuch verstanden, das auf das Fremdsprachenlernen bezogen ist. Umstritten ist die Frage, wie diese Berücksichtigung der "Faktorenkomplexion Fremdsprachenunterricht" (Kleineidam/Raupach 1995: 298) erfolgt bzw. erfolgen soll, wovon wiederum die Mehrdeutigkeit des Begriffs didaktische Grammatik abhängt. In dem hier gegebenen Rahmen wird auf eine ausführliche Auseinandersetzung mit der Problematik der Begriffsbestimmung verzichtet; ${ }^{3}$ verweilen möchte ich jedoch auf der Verbindung didaktische Grammatik-Fremdsprachenunterricht, die aus meiner Sicht relevant ist.

Diese Verbindung legt es nahe, die Bezeichnung didaktische Grammatik mit dem Terminus Fremdsprachengrammatik (cf. Kleineidam/Raupach 1995) synonym zu verwenden. Dabei muss jedoch darauf geachtet werden, dass den zwei Termini zum Teil unterschiedliche begriffsbestimmende Kriterien zugrunde liegen: Entscheidend für den ersten Begriff - didaktische Grammatik - sind in erster Linie außersprachliche Faktoren, die die Grammatikschreibung beeinflussen, wie z. B. psychologische, lernkognitive methodische Faktoren (cf. Helbig 1992: 138). Für den Begriff Fremdsprachengrammatik ist dagegen der Benutzerkreis, an den sich die Grammatik wendet (Lernende einer Fremdsprache eben), bestimmend. In diesem Sinne lässt sich didaktische Grammatik am besten in der Gegenüberstellung mit linguistischer Grammatik bestimmen und näher erläutern, während Fremdsprachengrammatik vor dem Hintergrund der Polarisierung "Grammatiken für den Muttersprachenunterricht" vs. "Grammatiken für den Fremdsprachenunterricht" (cf. Helbig 1992: 141) anzusehen ist. Es liegt auf der Hand, dass die zwei Aspekte eng miteinander verbunden sind und sich zum Großteil decken; aus rein terminologischer Sicht wirkt die Synonymie jedoch etwas einengend. Der Begriff didaktische Grammatik lässt sich vielmehr als Oberbegriff betrachten, im Rahmen dessen sowohl Grammatiken für den Muttersprachenunterricht als auch für den Fremdsprachenunterricht anzusiedeln sind. Hier können und sollen wiederum weitere Differenzierungen vorgenommen werden, ausgehend von der folgenden von Helbig formulierten Grundannahme: "Eine MSG [= Grammatik für den Muttersprachenunterricht] ist vornehmlich auf die Systematisierung und Bewusstmachung sprachlicher Regeln gerichtet, über die der Muttersprachler auf Grund seiner sprachlichen Kompetenz (seines 'Sprachgefühls') bereits verfügt [...]. Während eine MSG von der Kompetenz ausgehen und sich auf sie stützen kann, muß eine FSG [= Grammatik für den Fremdsprachenunterricht] (da dem Lernenden zunächst die Kompetenz in der zu erlernenden Fremdsprache fehlt) dazu beitragen - als ein Mittel neben anderen, die Kompetenz in der Fremdsprache erst aufzubauen" (Helbig 1992: 141).

Gerade vor diesem Hintergrund kommt die Problematik der grammatischen Terminologie sehr deutlich zutage. Die Problematik stellt sich natürlich für didaktische Grammatiken im Allgemeinen, unabhängig davon, ob diese für den Muttersprach- oder Fremdsprachenunterricht konzipiert werden. In Fremdsprachengrammatiken gewinnt sie jedoch andere Dimensionen, da in dem Fall auch die herkömmliche grammatische Terminologie ins Spiel kommt.

Damit in Verbindung steht auch eine weitere Differenzierung im Rahmen der Kategorie Fremdsprachengrammatik, nämlich die nach der Berücksichtigung der Ausgangssprache der Zielgruppe. Demnach kann zwischen Grammatiken "mit Ausrichtung auf eine spezifische Grundsprache" und Grammatiken "ohne Ausrichtung auf eine spezifische Grundsprache"

\footnotetext{
${ }^{3}$ Verwiesen sei hierbei auf Götze (1999; 2001) und Helbig (1999; 2001).
} 
unterschieden werden (Kleineidam 1986: 28). ${ }^{4}$ Die meisten der hier berücksichtigten Grammatiken gehören der ersten Gruppe an; es sind also didaktische Grammatiken, die sich an Lernende mit Italienisch als Ausgangssprache wenden.

Das Kriterium der Ausrichtung auf eine bestimmte Sprache kann und muss (auch gerade hinsichtlich der Frage nach der grammatischen Terminologie) noch weiter ergänzt werden. Die Berücksichtigung der so genannten L1-Sprache der Adressaten kann sich nämlich auf die Verwendung dieser Sprache im Erklärungsteil - also auf die metasprachliche Ebene beschränken oder sie kann auch auf anderen Ebenen Niederschlag finden, allen voran in der Beschreibung sprachlicher Phänomene aus kontrastiver Sicht. Im ersten Fall handelt es sich im Grunde um reine Übersetzungen der entsprechenden deutschsprachigen Versionen, die sich an Lernende des Deutschen als Fremdsprache im Allgemeinen richten (cf. Dreyer/ Schmitt 1993 und Reimann 1998). Im zweiten Fall wenden sich die Grammatiken nicht nur explizit an den DaF-Unterricht in Italien, sondern sie sind darüber hinaus ausschließlich für diesen Benutzerkreis konzipiert. Die Mehrheit der hier zitierten Grammatiken lässt sich dieser zweiten Gruppe zuordnen.

\section{Grammatische Terminologie aus lerntheoretischer Sicht}

Aus lerntheoretischer Sicht wird dem Aspekt der grammatischen Terminologie eine grundlegende Bedeutung beigemessen, ausgehend von der Annahme, dass eine klare fachsprachliche Benennung von grammatischen Sachverhalten die Lernbarkeit und Behaltbarkeit derselben fördern kann (cf. Kleineidam 1986). Nützlich scheinen mir in diesem Zusammenhang die Überlegungen von Heringer/Keller-Bauer (1984), denen zufolge die Forderung nach terminologischer Regulierung anhand von drei Grundprinzipien erfüllt wird: das Verständlichkeitsprinzip, das Anschlussprinzip, das Anpassungsprinzip.

Das Prinzip der Verständlichkeit verweist auf die Notwendigkeit, eine klare Definition des jeweiligen Terminus anzugeben, damit sich der Benutzer über den gemeinten Begriffsinhalt und -umfang im Klaren ist. Unter Anschluss ist die Anschließbarkeit einzelsprachlicher Grammatiken gemeint; es geht also darum, dem Lernenden durch eine interlinguale Harmonisierung fachsprachlicher Termini den Übergang von einer zur anderen Grammatik zu erleichtern (bzw. zu ermöglichen). Das dritte Prinzip - Anpassung - verweist darauf, dass terminologische Entscheidungen vor dem Hintergrund der Merkmale und Bedürfnisse der Adressatengruppe erwogen werden sollten (Grundsprache, Alter, Lernziele usw.).

Wie verhalten sich unsere Grammatiken auf der terminologischen Ebene? Die durchgeführte Analyse stützt sich an Kleineidams (1986) grundsätzliche Unterscheidung zwischen "terminologischer Äquivalenz" und "terminologischer Divergenz": "Unter terminologischer 'Äquivalenz' verstehen wir Doppel- und Mehrfachbenennungen des gleichen Phänomens, die zueinander in einer Synonymrelation stehen. Unter terminologischer 'Divergenz' verstehen wir Unterschiede der Benennungen, die durch das zugrunde liegende Sprachbeschreibungsmodell oder durch strukturelle Verschiedenheiten der Sprachen bedingt sind" (Kleineidam 1986: 173).

Dass im interlingualen Vergleich Fälle von terminologischer Divergenz viel häufiger vorkommen, braucht nicht eigens erwähnt zu werden. Anhand zweier Beispiele soll dem Phänomen und dessen Folgen nachgegangen werden.

\subsection{Erster Beispielfall: Wortartenbenennung}

Das erste Beispiel von divergierender Terminologie betrifft den Bereich der Wortartenbenennungen. Die Divergenz ist in diesem Fall sowohl durch unterschiedliche, den Sprachbeschrei-

\footnotetext{
${ }^{4}$ Kursiv im Original.
} 
bungen zugrunde liegende Kategorisierungen und Begriffsbildungen als auch durch strukturelle Verschiedenheiten der Sprachsysteme bedingt.

Die deskriptive Klassifizierung der Wörter einer Sprache stellt bekanntlich ein sehr komplexes Gebiet dar, das selbst innerhalb einer Sprache zu unterschiedlichen Ergebnissen führen kann. Die Wortart-Terminologie ist demnach schon innerhalb einer Sprache uneinheitlich. Man denke beispielsweise an die in der deutschsprachigen Grammatikographie geläufigen Kategorisierungen im Bereich der Pronomina. Helbig/Buscha (2001: 320) bezeichnen als "Pronomina" nur die Wörter, die substantivisch (d.h. als Stellvertreter des Nomens) verwendet werden. Die adjektivisch gebrauchten Pronomina (Demonstrativ-, Possessiv-, Interrogativ- und Indefinitpronomina) werden dagegen der Klasse der "Artikelwörter" zugerechnet. Die Duden-Grammatik griff bis zu ihrer vorletzten Ausgabe auf die Kategorie der "Begleiter und Stellvertreter des Substantivs" zurück, innerhalb derer zwischen "Pronomina" und "Artikel" unterschieden wurde. Der Terminus Pronomen bezeichnet dabei den substantivischen als auch den adjektivischen Gebrauch der betreffenden Wörter, während der Artikel gemäß der traditionellen Auffassung in den bestimmten und den unbestimmten Artikel unterteilt wird (cf. Dudenredaktion 1998). Anders verfährt dagegen die letzte, neu erarbeitete Auflage (cf. Dudenredaktion 2005), die sich der Doppelbezeichnung "Artikelwörter und Pronomen" bedient. Der Ausdruck Artikelwort wird hier in Anlehnung an Helbig/Buscha verwendet (cf. Dudenredaktion 2005: 256).

Je nach Begriffsbildung kann man also in der deutschen Grammatiktradition den Artikel bzw. das Pronomen im engeren Sinne und den Artikel bzw. das Pronomen im weiteren Sinne unterscheiden. Dies entspricht nicht der italienischen (und überhaupt der romanischen) Grammatiktradition, in der sowohl der Begriff des Artikels als auch der des Pronomens eng gefasst werden: als "articoli" gelten demnach die "articolo determinativo", "articolo indeterminativo" und "articolo partitivo", während als "pronomi" nur die substantivisch gebrauchten Pronomina betrachtet werden. Der Gebrauch vor Nomen wird dagegen mit der Bezeichnung "aggettivo" abgedeckt. Die "possessivi", "dimostrativi", "indefiniti", "interrogativi" sind also - je nach syntaktischem Kontext - entweder "pronomi" oder "aggettivi".

Die letzt genannte Unterscheidung zwischen "pronomi" und "aggettivi" wird auch in den deutschen Grammatiken beibehalten, die sich an Italiener wenden. In anderen Worten: Eine aus der italienischen Grammatikschreibung stammende Differenzierung wird auf die Beschreibung des Deutschen übertragen. So unterscheiden z. B. fast alle hier berücksichtigte Grammatiken die "pronomi possessivi" und die "aggettivi possessivi" (cf. Difino/Fornaciari 1999; Bruno/Franch 1991; Seiffarth/Medaglia 1998). Weerning/Mondello (1996) finden eine andere Lösung, indem sie vom Oberbegriff "possessivi" ausgehen und dann auf die terminologische Differenz zwischen der deutschen und italienischen grammatischen Terminologie verweisen. Auf die italienische Terminologie rekurriert auch die Übersetzung von Dreyer/ Schmitt (1999); in dem Fall wird also in den Originaltext eingegriffen, um ihn mit der zielsprachlichen Grammatiktradition in Einklang zu bringen.

Die in Deutschland verfassten didaktischen Grammatiken bedienen sich der deutschen Terminologie: So wird der Begriff der Pronomina in Dreyer/Schmitt (1996) und in Hall/Scheiner (1997) weit gefasst, während Fandrych/Tallowitz (2000) und Reimann (1996) ${ }^{5}$ den Terminus "Artikelwort" wählen, wodurch die Domäne des Pronomenbegriffes eingeengt wird.

Das Beibehalten der italienischen Terminologie in den für Italienisch sprechende Lernende konzipierten Grammatiken kommt gewiss der Auffassung entgegen, dass fremdsprachengrammatische Terminologie das Erlernen der Fremdsprache nicht zusätzlich erschweren

\footnotetext{
${ }^{5}$ In der entsprechenden italienischen Übersetzung (Reimann 1998) wird "Artikelwort" mit "articolo e i suoi sostituti" wiedergegeben.
} 
sollte. $\mathrm{Zu}$ erwägen wäre meines Erachtens jedoch, ob die gelegentliche Einführung neuer Termini bzw. die Erweiterung der schon vorhandenen didaktisch auch sinnvoll und nützlich sein kann. Im Fall von "Artikelwort" könnte dies der Fall sein, da durch diese Wortartbezeichnung gewisse Merkmale der dazugehörenden Wörter signalisiert werden, die gerade im deutsch-italienischen Vergleich relevant sind. In Anlehnung an Helbig/Buscha (2001: 320f.) fasse ich diese Merkmale wie folgt:

a) Die Artikelwörter stehen immer vor einem Substantiv, wobei zwischen den beiden auch andere Wörter stehen können;

b) Mit einem Artikelwort kann kein anderes Artikelwort verbunden werden;

c) Das Artikelwort kann seine Position im Satz nicht alleine, sondern nur zusammen mit dem zugehörigen Substantiv ändern;

d) Zwischen dem Artikelwort und dem zugehörigen Substantiv besteht Kongruenz in Genus, Kasus und Numerus;

e) Die Artikelwörter determinieren den Deklinationstyp des folgenden Adjektivs;

f) das Auftreten der Artikelwörter ist obligatorisch (das gilt auch für den Nullartikel).

Aus der Perspektive der Italienisch sprechenden Deutschlerner erweisen sich die unter b), d), e) (und manchmal auch a)) angegebenen Merkmale als bedeutend, da hier strukturelle Unterschiede mit dem Italienischen bestehen und daher Interferenzfehler häufig sind. In den hier zitierten Grammatiken für Italiener wird tatsächlich v. a. auf den Punkt b) kontrastiv eingegangen, nämlich darauf, dass vor dem Artikelwort, z. B. dem Possessivartikel, kein anderer Artikel stehen kann. Die Vermutung, dass die Bezeichnung "articolo possessivo" (statt "aggettivo possessivo") die italienischen Lernenden auf die Besonderheiten der Wortklasse im Deutschen hinweisen könnte, liegt nahe. Diesbezüglich machen Lieber/Listl (1991: 76) den folgenden Vorschlag: "Da im Italienischen alles das, was außer dem bestimmten und unbestimmten Artikel im Deutschen zu den Artikelwörtern zählt, unter dem Oberbegriff aggettivi determinativi subsumiert wird [...], bietet es sich an, den von der DVG [= Dependenzverbgrammatik] vorgeschlagenen Begriff als determinativi für alle Artikelwörter zu übernehmen. Pronomen bleiben dann nur die eigentlichen Prowörter."6

Bezieht man den Begriff "determinativi" auf das italienische "articolo determinativo", so werden die oben erwähnten Merkmale der Artikelwörter im Deutschen nicht signalisiert; in diesem Sinne erscheint die Bezeichnung "articolo e i suoi sostituti" (Reimann 1998) als geeigneter.

\subsection{Zweiter Beispielfall: Satzgliederbenennung}

Das zweite Beispiel betrifft die Ebene der Satzglieder, ein Gebiet, auf dem innerhalb der Grammatiken (und allgemein in den Lehrwerken) für Deutsch als Fremdsprache die Grundkategorien der valenztheoretischen Ansätze große Verbreitung gefunden haben. Diese haben sich bekanntlich als besonders geeignet erwiesen, um Einsicht in den Satzbauplan des deutschen Satzes zu gewinnen. Götze (1999: 139) weist diesbezüglich darauf hin, dass die Stärke des valenzorientierten Beschreibungsmodells vor allem in den folgenden Bereichen liegt: syntaktische und semantische Beschreibung der Umgebung des Verbs als des angenommenen Zentrums des Satzes; Unterscheidung valenzabhängiger und valenzunabhängiger Teile im Satz; Klärung zahlreicher Probleme der traditionellen Schulgrammatik (z. B. Unterscheidung der adverbialen Bestimmungen und präpositionalen Objekte; die Stellung der Negationspartikel nein usw.); Klärung von schwierigen Detailproblemen der Satzgliedstellung, insbesondere im Mittelfeld des Satzes. Außerdem eignet sich die dependenz-/valenz-

\footnotetext{
${ }^{6}$ Auf diese terminologische Lösung wird in der Grammatik von Saibene (1992: 126) zurückgegriffen.
} 
orientierte Sprachbeschreibung für ein kontrastives Vorgehen, wodurch nicht nur das Erlernen der Fremdsprache sondern auch das Verstehen der Muttersprache gefördert wird.

Das valenzorientierte Beschreibungsmodell findet auch in der Terminologie der hier untersuchten DaF-Grammatiken Niederschlag. Dabei fällt auf, dass diese Terminologie oft mit den traditionellen Fachtermini vermischt wird. Dies betrifft in erster Linie die begrifflichen Oppositionen Ergänzung - Angabe bzw. Objekt - Adverbiale, die z. T. synonym verwendet bzw. als deckungsgleich betrachtet werden, woraus sich gelegentlich auch terminologische Uneinheitlichkeiten ergeben. Dazu einige Beispiele.

Fandrych/Tallowitz (2000) verwenden auf der Satzgliedebene die Termini "Objekte", "Adverbien" und "Angaben". Diese Differenzierung ist aus mehreren Gründen problematisch. Zunächst einmal, weil "Adverb" eine wortart- und nicht eine satzgliedbezogene Kategorie ist. D.h., in dem Fall müsste man eigentlich von "Adverbialen" sprechen. Dies wäre aber in diesem Zusammenhang dennoch falsch, da die "Adverbien" in Fandrych/Tallowitz (2000) nicht ganz mit der Kategorie der Adverbialbestimmungen übereinstimmen. Der Terminus bezeichnet nämlich nur die durch Adverbien repräsentierten Satzglieder, die vom Verb her vorgesehen sind, d. h. adverbiale Ergänzungen (z. B. "Links ist meine Schwester." (Fandrych/ Tallowitz 2000: 58), "Ich möchte am liebsten irgendwohin fahren.", "Schau mal nach oben!" (Fandrych/Tallowitz 2000: 68)), während die freien Satzglieder als "Angaben" gelten. Sowohl "Adverbien" als auch "Angaben" werden nach semantischen Gesichtspunkten differenziert: im ersten Fall unterscheiden die Autoren "Lokal-Adverbien " und "Direktional-Adverbien", im zweiten die "Temporal-, Kausal-, Final- und Modal-Angaben". Eine semantische Differenzierung findet auch innerhalb der Kategorie der Objekte statt, der folgende Satzglieder zugeordnet werden: "Akkusativ-objekt", "Dativ-objekt", "Direktional-objekt", "Lokal-objekt", "Präpositional-objekt" und "Objekt mit Präposition", wobei die Kriterien für die Unterscheidung zwischen Lokal- bzw. Direktionalobjekten und Lokal- bzw. Direktionaladverbien nicht immer nachvollziehbar sind. Laut den angegebenen Erklärungen beruht sie auf dem Kriterium der morphologischen Repräsentation der Satzglieder: die "Lokal- und Direktionalobjekte" werden durch eine Präposition eingeleitet, während die Realisierung der entsprechenden Adverbien durch reine Adverbien erfolgt (s. z. B. "Hinter uns stehen meine Eltern." vs. "Hinten stehen meine Eltern" (Fandrych/Tallowitz 2000: 58)). Dies widerspricht jedoch der Tatsache, dass unter den Beispielen für "Lokal- bzw. Direktional-Adverbien" auch präpositional eingeleitete Adverbien auftauchen (cf. den oben angeführten Beispielsatz "Schau mal nach oben!").

Auch Dreyer/Schmitt (1996: 127-133) sprechen einerseits von "Objekten" (Dativobjekt, Akkusativobjekt, Präpositionalobjekt bzw. präpositionales Objekt) und andererseits von "Angaben", die semantisch klassifiziert werden. Der Begriff "Angabe" hat hier aber einen weiteren Umfang als in der Opposition "Ergänzung" vs. "Angabe". Er bezieht sich nämlich auf die traditionellen Adverbialen, die sowohl Angaben als auch Ergänzungen sein können. So finden sich unter den Beispielen für adverbiale Angaben die Sätze "Er kommt jeden Tag um acht Uhr." und "Er wohnt in der Gartenstraße neben dem Postamt." (Dreyer/Schmitt 1996: 337). ${ }^{7}$ Bei den Objekten wird eine weitere Unterscheidung nach der Wortart vorgenommen, der zufolge die Objekte, die in Form von Pronomen auftreten, als eine separate Kategorie behandelt werden. Dabei handelt es sich um eine ziemlich ungewöhnliche Differenzierung, die in dem gegebenen Zusammenhang auch insofern fraglich ist, als in diesem Fall

\footnotetext{
${ }^{7}$ Es wird ferner auch zwischen adverbialen und präpositionalen Angaben unterschieden: als Beispiel für die "Satzstellung mit adverbialen und präpositionalen Angaben" wird z. B. der Satz "Ich komme morgen mit Vergnügen zu eurer Party." angegeben, während unter "Satzstellung mit Objekten und adverbialen Angaben" der Satz "Ich schreibe morgen meinem Mann wegen der Sache einen Brief nach Italien" zu finden ist (Dreyer/ Schmitt 1996: 131). Es handelt sich jedoch um eine meines Erachtens für die Grammatikbenutzer schwer nachvollziehbare Differenzierung.
} 
die entsprechenden Objekte als "Akkusativpronomen" bzw. "Dativpronomen" bezeichnet werden.

Reimann (1996) differenziert "Ergänzungen" und "Angaben". Zu den Ergänzungen gehören das Subjekt, das Dativobjekt, das Akkusativobjekt und das Präpositionalobjekt (auch "Ergänzung im Dativ/Akkusativ mit Präposition" genannt). Bei den Angaben werden die häufig vorkommenden semantischen Klassen zitiert (temporale, kausale, modale und lokale Angaben).

Hall/Scheiner (1997: 278) gehen von "Objekten" aus und definieren sie als "[...] - je nach Verb - obligatorische oder fakultative Ergänzungen zum Prädikat" und unterscheiden innerhalb der Kategorie die Kasusobjekte (Akkusativ-, Dativ- und Genitivobjekt) und die Präpositionalobjekte. Die freien Satzglieder werden allgemein "adverbiale Angaben" genannt (und diese weiter nach dem semantischen Wert unterteilt). Die Klasse der adverbialen Angaben wird samt den adverbialen Ergänzungen mit der Klasse der adverbialen Bestimmungen gleichgesetzt.

In Grammatiken, die sich explizit an Italienisch sprechende Lernende wenden, muss diese bereits auf intralingualer Ebene durchaus heterogene Terminologie der Satzstrukturbeschreibung auch vor dem Hintergrund der in der italienischen Grammatikographie geläufigen Fachbezeichnungen erwogen werden.

Lieber/Listl (1991: 78) betonen zu Recht, dass es für Italiener grundsätzlich keinen großen Unterschied macht, ob man der traditionellen Einteilung in Objekte und Adverbialbestimmungen oder der jüngeren in Ergänzungen und Angaben folgt. Aus ihrer eigenen Grammatik ist ihnen nämlich die Unterscheidung, die diesen Begriffspaaren zugrunde liegt, nicht bekannt, da die italienische Grammatik den Satz traditionell mit Hilfe der Begriffe "soggetto", "predicato" und "complementi" beschreibt. Diese entstammen der so genannten "analisi logica", der zufolge die Satzteile in erster Linie als Träger bestimmter semantischer Rollen betrachtet werden. Die so abgegrenzten Satzglieder werden daher nach semantisch-logischen Kriterien differenziert, die gelegentlich mit syntaktischen vermischt werden.

Nach Dardano/Trifone (1997: 122) sind die "complementi [...] i vari componenti della frase che hanno la funzione di completare quanto è espresso dai due componenti fondamentali, soggetto e predicato"8; das Wort completare 'ergänzen' ist hier also kein Fachterminus im Sinne der Dependenzverbgrammatik. Die "complementi" werden traditionell in "complemento diretto" ("complemento oggetto") und "complementi indiretti" unterteilt. Im ersten Fall überschneiden sich semantische (das unmittelbare Betroffensein) und syntaktische Gesichtspunkte (die enge syntaktische Beziehung zum Verb). Bei der Definition der "complementi indiretti" spielt auch das Kriterium der morphologischen Repräsentation eine Rolle: Traditionell werden nämlich als "complementi indiretti" die von einer Präposition eingeleiteten Satzglieder definiert. Dieses Kriterium erweist sich jedoch manchmal als irreführend, da es auch uneingeleitete "complementi indiretti" gibt (man denke z. B. an den Satz "Camminava lentamente."; cf. Marotta 1996: 156). Innerhalb der Kategorie werden weiters - vorwiegend nach dem semantischen Wert - zahlreiche Klassen differenziert. Die valenzorientierte Perspektive ist offensichtlich in dieser Begriffsbildung nicht vorhanden, wodurch sich die Deckung mit den Begriffspaaren Objekte/Adverbiale bzw. Ergänzungen/Angaben als schwierig erweist.

In den hier betrachteten Grammatiken finden sich verschiedene terminologische Lösungen. Bruno/Franch (1991: 4f.) bezeichnen - ausgehend von morphologischen und syntaktischen Charakteristiken - als "complementi diretti" die kasusbestimmten und uneingeleiteten Satzglieder, während die, die präpositional eingeleitet oder durch Adverbien repräsentiert werden,

\footnotetext{
${ }^{8}$ Die verschiedenen Satzteile, die die Funktion haben, das von den zwei Hauptsatzteilen, dem Subjekt und dem Prädikat, Ausgedrückte zu ergänzen.
} 
die Klasse der "complementi indiretti" und "avverbi" bilden. Hier werden aber (wie schon bei Fandrych/Tallowitz der Fall war) zwei Beschreibungsebenen vermischt. In Difino/Fornaciari (1999: 207) wird "complemento diretto" wieder im weiten Sinne verwendet (also in Bezug auf alle kasusmarkierten Satzglieder), ${ }^{9}$ während man die "complementi indiretti" in "complementi di tipo avverbiale o preposizionale" unterteilt. Folgt man dieser Einteilung, würde sich der Terminus "complemento con preposizione" (auch "complemento preposizionale") sowohl mit den von einer Präposition eingeleiteten Ergänzungen als auch mit den ebenfalls durch präpositionale Fügungen repräsentierte - Adverbialbestimmungen decken (cf. Difino/Fornaciari 1999: 207: "Der Student steht mit einem Rucksack am Bahnhofseingang.").

Bei diesen Einteilungen fallen meistens die Präpositionalobjekte aus, d. h. man unterscheidet im Grunde nicht - zumindest nicht terminologisch - zwischen den (meist fakultativen) Adverbialen, die durch Präpositionen eingeleitet werden und den vom Verb verlangten Präpositionalobjekten. Gerade diese Unterscheidung ist aber äußerst wichtig für den Deutschlerner: Bei Verben, die ein Präpositionalobjekt verlangen, ist es nämlich nötig, die Präposition quasi als Teil des Verbs zu betrachten (und sie samt dem Verb zu lernen), da sie vom Verb bestimmt ist und ihre ursprüngliche Bedeutung nicht mehr hat. Anders dagegen bei präpositionalen Adverbialen, wo die Präposition je nach semantischem Wert des Verbs gewählt werden kann (und muss). Es wäre also m. E. wichtig, dieses Satzglied auch in den auf Italienisch verfassten Grammatiken des Deutschen mit einem entsprechenden Terminus zu bezeichnen und ihn bei der Beschreibung des Satzes zu behandeln. Saibene (1992: 235) wählt dabei "complemento retto da preposizione", während Weerning/Mondello (1996: 114) von "complemento introdotto da preposizione" sprechen, wobei die letzte Bezeichnung irreführend sein kann, da es - nach morphologischen Kriterien betrachtet - zahlreiche "complementi introdotti da preposizione" gibt. ${ }^{10}$

Damit haben wir aber noch nicht den Aspekt der Abhängigkeit bzw. Unabhängigkeit der "complementi" vom Verb terminologisch erfasst, da, wie gesagt, diese Unterscheidung im Begriff nicht implizit ist. Die entsprechenden italienischen Termini für die Tesnierschen actants und circonstants sind "attanti" und "circostanziali" (cf. Dardano/Trifone 1997: 111). Diese finden jedoch in didaktischen Grammatiken kaum Verwendung, auch nicht in den für den muttersprachlichen Italienischunterricht. Als Beispiel sei hier auf die nach valenztheoretischen Ansätzen konzipierte Grammatik von Corti/Caffi (1989) verwiesen: In dieser Grammatik wird der Terminus argomenti als Oberbegriff für complementi verwendet. Zu den "argomenti" zählt hier auch das Subjekt, das entsprechend dem valenzorientierten Ansatz seine Hauptrolle aufgibt. Unter den "complementi" wird aber - bis auf traditionelle Abgrenzungen nach semantischen Gesichtspunkten - keine weitere Differenzierung vorgenommen, so dass das Problem ungelöst bleibt. Lieber/Listl (1991: 79-81) plädieren diesbezüglich für eine Unterscheidung zwischen "complementi obbligatori" (Ergänzungen) und "complementi facoltativi" (Angaben), da sie es aus didaktischen Gründen für sinnvoll halten, auf die Einführung einer völlig neuen Kategorie zu verzichten. Gegen dieses Argument lässt sich jedoch einwenden, dass durch die Bezeichnung obligatorisch das Kriterium des obligatorischen syntaktischen Vorkommens dieser Elemente an der Oberfläche suggeriert werden kann. Dann wäre sie nämlich irreführend. Darauf machen Weerning/Mondello (1996: 284) aufmerksam, indem sie die deutschen Termini Ergänzungen und Angaben als Oberbegriffe

\footnotetext{
${ }^{9}$ So auch bei Saibene 1992.

${ }^{10}$ In der zitierten Grammatik besteht die Gefahr jedoch nicht, weil fast alle anderen "complementi" semantisch differenziert werden. Andere Grammatiken (cf. Difino/Fornaciari 1999) enthalten nur Listen von "verbi che reggono preposizioni". Eine sehr fragwürdige Bezeichnung findet sich in Bruno/Franch (1991: 332-343), die von "Prädikatsergänzungen" sprechen.
} 
beibehalten und im Rahmen der Ergänzungen die "complementi di tipo obbligatorio" von den "complementi di tipo facoltativo" trennen. ${ }^{11}$

\section{Abschließende Bemerkungen}

Ziel dieses Beitrags war es, die grammatische Terminologie in den im italienischen DaFUnterricht meist verwendeten Grammatiken zu untersuchen. Der Hauptschwerpunkt liegt dabei auf der Gegenüberstellung der grammatischen Terminologie im Deutschen und im Italienischen. Durch die zwei dargestellten Beispiele wurden insbesondere Benennungen in den Bereichen Wortarten- und Satzgliedanalyse betrachtet. Aus der Analyse wird ersichtlich, dass grammatische Termini auf interlingualer Ebene sehr oft divergieren und dass diese Divergenz sowohl an den strukturellen Verschiedenheiten der Sprachsysteme als auch an den unterschiedlichen theoretischen Ansätzen liegt, nach denen die Sprachbeschreibung erfolgt. Terminologien spiegeln somit auch Besonderheiten einer Sprache wider.

Dadurch stellt sich die Frage, ob und inwieweit die Forderung nach einer interlingualen Harmonisierung fachsprachlicher Termini - nach dem oben genannten Prinzip der Anschließbarkeit $-\mathrm{zu}$ vertreten ist. Eine solche Forderung scheint vielmehr im einzelsprachlichen Bereich Geltung zu haben, während der Bereich der Fremdsprachengrammatikschreibung mit der Herausforderung konfrontiert wird, Divergenz terminologisch und begrifflich verständlich zu machen. Dies stellt wiederum weitere Anforderungen an den Unterricht, allen voran die nach dem "Aufbau einer Grammatikendidaktik"12 (Hennig 2001: 9) im Schul- wie im Hochschulbereich. Gemeint ist damit eine Schulung der Lernenden im Umgang mit Grammatiken, grammatischen Aussagen und grammatischer Terminologie.

\section{Literaturangaben}

Bianco, Maria Teresa (1996): Valenzlexikon Deutsch-Italienisch. Dizionario della valenza verbale. 2 Bde. Heidelberg.

Bruno, Elena/Franch, Raffaela (1994): Deutsche Grammatik. Grammatica di riferimento per lo studio della lingua tedesca. 3. Aufl. Torino.

Corti, Maria/Caffi, Claudia (1989): Per filo e per segno. Grammatica italiana per il biennio. Milano.

Dardano, Maurizio/Trifone, Pietro (1997): La Nuova Grammatica della lingua italiana. Bologna.

Difino, Elisabetta/Fornaciari, Paola (1999): Tipps. Milano.

Difino, Elisabetta/Fornaciari, Paola (2001): Deutsch Klipp und Klar! Milano.

Dreyer, Hilke/Schmitt, Rainer (1996): Lehr- und Übungsbuch der deutschen Grammatik. Neu bearb. Ausgabe. Ismaning.

Dreyer, Hilke/Schmitt, Rainer (1999): Grammatica tedesca con esercizi. Tradotta e rielaborata da Simonetta Guarini e Luciana Ziglio. Ismaning.

Dudenredaktion (ed.) (1998): Duden Grammatik der deutschen Gegenwartssprache. 6., neu bearb. Aufl. Mannheim etc.

Dudenredaktion (ed.) (2005): Duden Grammatik der deutschen Gegenwartssprache. 7., völlig neu erarb. und erw. Aufl. Mannheim etc.

Fandrych, Christian/Tallowitz, Ulrike (2000): Klipp und Klar. Übungsgrammatik Grundstufe Deutsch. München.

\footnotetext{
${ }^{11}$ Es sei hier auch auf das deutsch-italienische Valenzwörterbuch von Bianco (1996) verwiesen und auf die dort vorgenommene terminologische Unterscheidung: Zunächst wird zwischen "complementi" (Ergänzungen) und "aggiuntivi" (Angaben) unterschieden und die ersten werden dann weiters in "complementi di verbo" (obligatorische Ergänzungen) und "complementi di verbo facoltativi" (fakultative Ergänzungen) unterteilt.

12 Kursiv im Original.
} 
Götze, Lutz (1999): "Eine funktionale Grammatik für Deutsch als Fremdsprache". In: Skibitzki, Bernd/Wotjak, Barbara (eds.): Linguistik und Deutsch als Fremdsprache. Festschrift für Gerhard Helbig zum 70. Geburtstag. Tübingen: 81-94.

Götze, Lutz (2001): "Linguistische und didaktische Grammatik". In: Helbig, Gerhard et al. (eds.): Deutsch als Fremdsprache. Ein internationales Handbuch. 1. Halbband. Berlin/ New York: 187-194.

Hall Karin/Scheiner, Barbara (1997): Übungsgrammatik Deutsch als Fremdsprache für Fortgeschrittene. 2. Aufl. Ismaning.

Helbig, Gerhard (1981): Sprachwissenschaft - Konfrontation - Fremdsprachenunterricht. Leipzig.

Helbig, Gerhard (1992): "Grammatiken und ihre Benutzer". In Ágel, Vilmos/Hessky, Regina (eds.): Offene Fragen - offene Antworten in der Sprachgermanistik. Tübingen: 135-150.

Helbig, Gerhard (2001): "Arten und Typen der Grammatik". In: Helbig, Gerhard et al. (eds.): Deutsch als Fremdsprache. Ein internationales Handbuch. 1. Halbband. Berlin/New York: 175-187.

Helbig, Gerhard/Buscha, Joachim (2001): Deutsche Grammatik: ein Handbuch für den Ausländerunterricht. Neu bearbeitete Ausgabe. Leipzig etc.

Hennig, Mathilde (2001): Welche Grammatik braucht der Mensch? Grammatikenführer für Deutsch als Fremdsprache. Unter Mitarbeit von Carsten Hennig. München.

Heringer, Hans Jürgen/Keller-Bauer, Friedrich (1984): "Probleme einer gebrauchsgrammatischen Terminologie". In: Sprache und Literatur in Wissenschaft und Unterricht 53: 6586.

Ivančić, Barbara (2003): Deutsche Sprache, schwere Sprache - Ma le grammatiche ci aiutano? Considerazioni sulle grammatiche didattiche del tedesco. Trieste.

Kleineidam, Hartmut (1986): Fremdsprachengrammatik: Analysen und Positionen. Beiträge mit dem Schwerpunkt Französisch. Tübingen.

Kleineidam, Hartmut /Raupach, Manfred (1995): "Grammatiken". In: Bausch, Karl-Richard et al. (eds.): Handbuch Fremdsprachenunterricht. 3. überarb. und erw. Aufl. Tübingen/Basel: 298-301.

Lieber, Maria/Listl, Verena (1991): "Eine optimale Grammatik für Italiener? Zur Adressatenspezifik im Ausländerunterricht oder: Grammatik zwischen DVG und Tradition". In: Fremdsprachenunterricht lehren und lernen (FLuL) 20: 72-84.

Marotta, Giovanna (1996): "Complemento". In: Beccaria, Gian Luigi (ed.): Dizionario di linguistica e di filologia, metrica, retorica. Torino: 155-156.

Reimann, Monika (1997): Grundstufen-Grammatik für Deutsch als Fremdsprache. 2. Aufl. Ismaning.

Reimann, Monika (1998): Grammatica di base della lingua tedesca. Tradotta da Luciana Kromberg Tomelleri. Ismaning.

Saibene, Maria Grazia (2002): Grammatica descrittiva della lingua tedesca. Roma.

Seiffarth, Achim/Medaglia, Cinzia (1998): Arbeitsgrammatik. Rapallo.

Weerning, Marion/Mondello, Mariano (2000): Dies und Das. Grammatica di tedesco con esercizi. Rapallo. 